\title{
Preferences and Trends in Management of Rhegmatogenous Retinal Detachment in Pakistan
}

\author{
Muhammad Amer Awan ${ }^{1}$, Javeria Muid ${ }^{2}$ \\ ${ }^{1}$ Department of Ophthalmology, Shifa International Hospital H-8/4 Islamabad \\ ${ }^{2}$ Royal Victoria Eye and Ear Hospital, Dublin Ireland
}

\begin{abstract}
Purpose: To report the preferences and trends in managing Rhegmatogenous retinal detachment (RRD) in Pakistan.
\end{abstract}

Study Design: Cross sectional survey.

Place and Duration of Study: Shifa International Hospital, Islamabad, from December 2018 to January 2019.

Method: An online survey was conducted in which the practicing vitreoretinal (VR) surgeons, who were registered with vitreoretinal society of Pakistan were included. They were asked to respond to 10 questions to assess their practice and management strategies in treating RRD. The survey included general questions regarding their primary practice, preference of anaesthesia and type of vitrectomy machine they used and specific questions consisting of different scenarios of RRD.

Results: Sixty-two VR surgeons of Pakistan responded to this survey. Most of the VR surgeons belonged to Punjab (56\%) followed by Sindh (25\%). Regarding their primary practice setting $50 \%$ of VR surgeons worked both in government and private practice, $30 \%$ practiced in academic/university hospital and $20 \%$ of them had only private practice. Seventy percent of VR surgeons in Pakistan preferred local anaesthesia. In non-posterior vitreous detachment (PVD) RRD, majority (69\%) performed segmental buckling (SB) with or without encirclement. In pseudophakic superior macula on RRD with a single retinal tear $50 \%$ preferred pars plana vitrectomy (PPV) followed by SB in $25 \%$ and pneumatic retinopexy in $18 \%$. In inferior macula off RRD with a retinal tear at 70 'clock position, $56 \%$ of the VR surgeons performed PPV alone or combined with SB.

Conclusion: There is an increased trend towards PPV as a primary procedure for RRD in Pakistani VR surgeons. Local anaesthesia is the preferred anesthesia.

Key Words: Rhegmatogenous retinal detachment, Retinal break, Pars Plana Vitrectomy, Pneumatic Retinopexy.

How to Cite this Article: Awan MA, Muid J. Preferences and Trends in Management of Rhegmatogenous Retinal Detachment in Pakistan. Pak J Ophthalmol. 2021, 37 (1): 24-28.

Doi: https://doi.org/10.36351/pjo.v37i1.1157

\section{INTRODUCTION}

Rhegmatogenous retinal detachment (RRD) is a sight

Correspondence: Javeria Muid

Royal Victoria eye and ear hospital, Dublin Ireland

Email: javeriamuid90@hotmail.com

Received: November 3, 2020

Accepted: November 26, 2020 threatening retinal condition that requires urgent management and can lead to blindness if left untreated. ${ }^{1,2}$ Prevalence of RRD is from 6.3 to 17.9 per 100,000 people per year and has a lifetime risk of $0.06 \%$ approximately. ${ }^{3,4}$ There are various conditions that can lead to rhegmatogenous retinal detachment such as, tractional force of posterior vitreous detachment (PVD) that produces a retinal tear, allowing the fluid to access the sub retinal space through the break. ${ }^{5}$ Various predisposing factors are increasing age, previous cataract surgery, diabetes, 
blunt ocular trauma and myopia., ${ }^{6,8,9}$ The main aim of treatment is to identify the retinal breaks, seal them (with laser photocoagulation/cryopexy) and release any traction on the edges of the breaks. ${ }^{10}$ Various treatment modalities are available for this purpose for example, pneumatic retinopexy (PR), scleral buckle (SB), pars plana vitrectomy (PPV) and combined SB and PPV. ${ }^{11,12}$ These approaches are used throughout the world with the primary success rate of around $90 \% .{ }^{4} \mathrm{PPV}$ is reported to be the most common method of RRD repair. A study showed the primary reattachment rate of $95.6 \%$ with $27 \mathrm{~g}$ PPV for primary RRD. $^{12}$

The purpose of this survey was to report the current trends and preferences in managing different types of RRD in Pakistan. We also aim to compare our national management trends with international standards.

\section{METHODS}

Institutional review board and ethics committee at Shifa Tameer-e-Millat University and Shifa International Hospital approved this study and the study was performed in accordance with the relevant guidelines and regulation.

This was an online cross-sectional survey that involved the vitreo-retinal (VR) surgeons of Pakistan. Duration of the survey was one month from $16^{\text {th }}$ December 2018 to $15^{\text {th }}$ January 2019. The practicing vitreoretinal (VR) surgeons, who were registered with vitreoretinal society of Pakistan were included. They were asked to respond to 10 questions to assess their practice and management strategies in treating RRD. The survey included general questions regarding their primary practice, preference of anaesthesia and type of vitrectomy machine they used and specific questions consisting of different scenarios of RRD and VR surgeons were asked to give their opinions in that specific scenario. The data was collected and analyzed using Microsoft excel version 2016. Qualitative variables were presented as frequency and percentages.

\section{RESULTS}

Sixty-two VR surgeons responded to the survey questionnaire in the specified time from different areas of Pakistan. Fifty-six percent VR surgeons (34 of 62) who responded to the survey belonged to Punjab, followed by Sindh with $25 \%$ (15 of 62) and Islamabad with $10 \%$ (6 of 62). Regarding their primary practice setting, half of the total VR surgeons worked in both government and private practice, one third (18 of 62) practiced in academic/ university hospitals while, one fifth of them (13 of 62) had only private practice. Furthermore, most of the surgeons preferred to perform RD surgery in local anaesthesia (70\%). Preferences about the type of vitrectomy machine being used by most of the surgeons revealed that Constellation by Alcon was the most popular choice (43\%), being used by 27 surgeons, followed by DORC Eva \& Associate in 12 out of 62 (28\%) VR surgeons in Pakistan.

When asked about the preferences of procedure in different types of RRDs. Graph 1 shows the preferred choice of procedure in a 25 years old male patient with non-PVD, macula on RD, with multiple retinal holes anterior to equator in supero-temporal quadrant. Segmental buckling was the preferred choice.

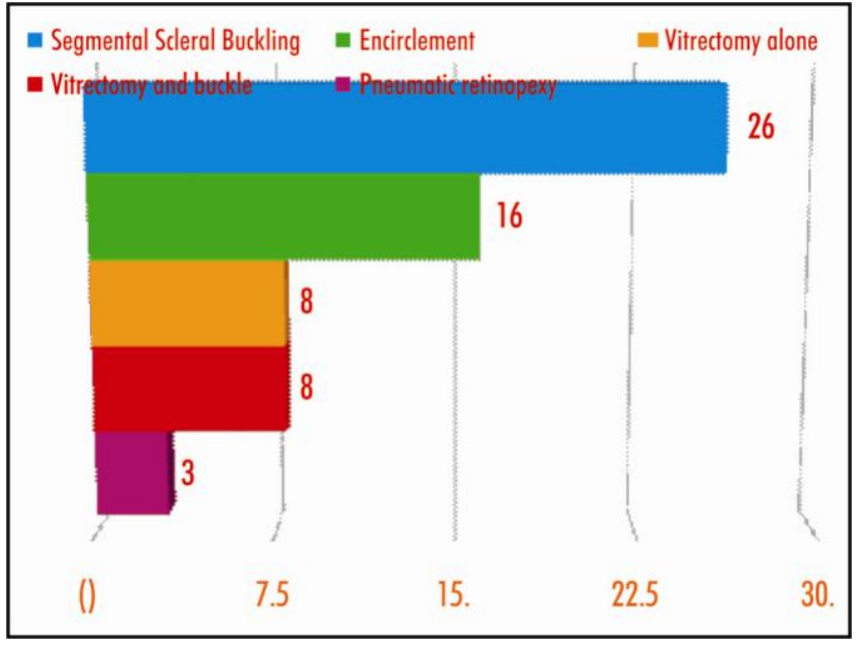

Fig. 1:

Second scenario included a patient with superior pseudophakic macula-on RD with a single retinal tear at 10 o' clock anterior to equator, in 65 years old female with -2.5 myopia. Graph 2 shows the response preference.

Third scenario was about the inferior macula-off RRD with a retinal tear at 7 O'clock in a 55-year old male. This question showed interestingly different results with no single popular choice by the VR surgeons. Graph 3. 


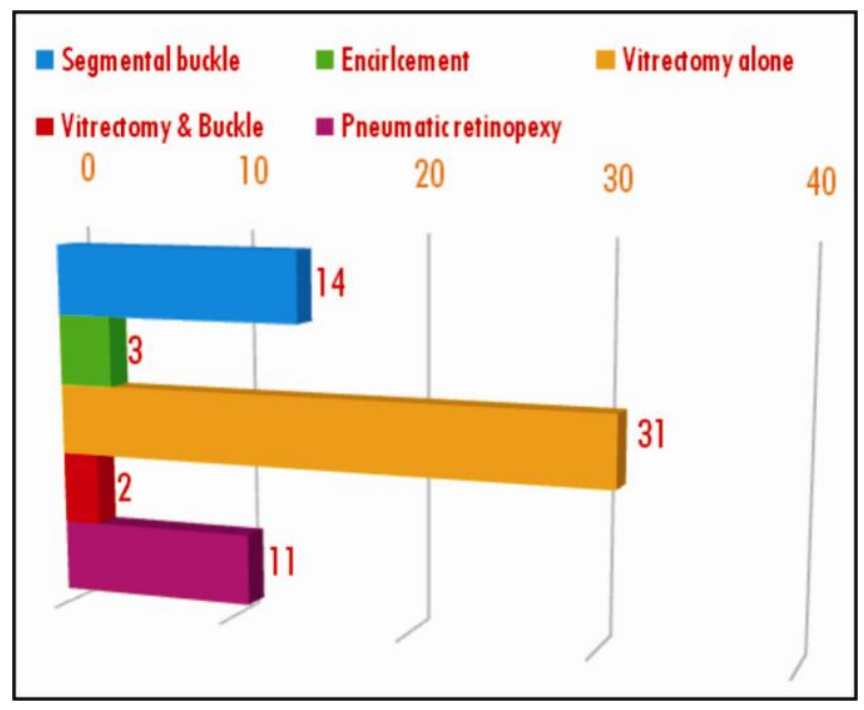

Fig. 2:



Fig. 3:

The results also showed that the trend of performing SB has decreased as $75 \%$ of the respondents were doing scleral buckling in only 11$20 \%$ of their patients with RRD. While $18 \%$ surgeons performed this procedure in $21-40 \%$ of their patients. Preferred choice of tamponade in PPV for RRD with retinal tear in superior half was gas, being chosen by $60 \%$ VR surgeons (26\% SF6, 26\%C3F8 and $8 \%$ C2F6) and $30 \%$ used 1000 centistokes silicone oil. In RRD with breaks in inferior half, $74 \%$ preferred silicone oil (1000 centistokes, 5000 centistokes or Densiron) and the remaining used medium (C2F6) or long acting gas $(\mathrm{C} 3 \mathrm{~F} 8)$.

\section{DISCUSSION}

In this study, we described the recent preferences and trends in the management of RRD in Pakistan. PPV is becoming the most popular choice to treat different types of RRD throughout Pakistan as well as internationally. It has gained worldwide popularity due to variety of reasons. Recent developments and advancements in mechanical and technical fields such as micro incision vitrectomy systems, high speed cutters, wide angle viewing systems and utilization of perfluorocarbon liquids led to better visibility and fewer complications in retinal reattachment surgery in comparison to the past decade. In modern training programs retinal surgeons are getting more exposure to PPV that has made them more comfortable with PPV than SB. On the other hand, the indications for PPV have been expanded to include spectrum of vitreo retinal diseases such as macular hole, epiretinal membrane and diabetic retinopathy whereas SB is only performed in RRD.

The appropriate treatment depends upon various factors such as: age of the patient, presence of PVD, complexity of detachment, whether breaks are anterior or posterior and surgeon's preference as well. ${ }^{13}$ In simple detachment SB, PR and PPV are options depending on PVD and complex detachments require internal surgical approach. ${ }^{14}$ A study in US, in which 12779 patients of RRD were evaluated, it was found that treatment approaches were not only decided on the basis of patient-level characteristics but physician variations also made a huge difference. Geographical variations least affected the management approach. ${ }^{15}$ In 2012 US Medicare survey showed that 74\%, 11\% and $15 \%$ of primary RRD were repaired by PPV, SB and PR respectively. ${ }^{16}$ The preferences and trends (PAT) survey of 2015 showed that $67 \%$ of the vitreoretinal surgeons placed SB in $11 \%$ of RRDs, while $24 \%$ placed SB in $41 \%$ RRDs. ${ }^{17}$ Fischer et al. did a survey in August 2018 and showed that the surgeons were less willing to perform SB when multiple co-factors were present. ${ }^{18}$ For example, if two adjacent retinal breaks were present which could still be treated with SB only approximately 57\% would perform SB while the rest would simply go for PPV. ${ }^{18}$ They also compared their results with a survey done in 2001 which showed that there was a marked increase in trend towards PPV even in pseudophakic eyes.

We also compared our results with the international studies. Regarding superior RRD, in 
Korean study $74 \%$ surgeons chose to do SB, $16 \%$ preferred PR and 10\% went for PPV in $2013 .{ }^{19}$ In American society of retina specialists survey, only $6 \%$ would do SB, $68 \%$ of them favored PPV followed by $\mathrm{PR}$ in $26 \%{ }^{20}$ Our survey has shown that PPV was the preferred choice in Pakistan. However, one fourth of the respondents would do SB in our study. Eibenberger et al also stated that from 2009 to 2015 there was an increased trend towards performing PPV in primary RRDs as is seen in our survey in the case of PVD related RRDs. ${ }^{20}$ Minihen et al. retrospectively compared RRD surgeries, performed 20 years apart, in a single center located in London, UK. ${ }^{21}$ They reported that $63 \%$ patients with primary RRD were treated by PPV in 1999; in contrast, only one case was managed by PPV in 1979 and 1980. In contrast to this, with latest developments in cutters, fluidics and adjuncts, there is marked shift towards vitrectomy in simple and complex RRD.

On comparison with international trends shown by international surveys, there are some similarities as well as few differences. Popularity of PR is decreasing in recent times even in the case of uncomplicated primary RRD that was previously considered as a good indication. PR has few advantages over other procedures i.e. PPV and SB. These include shorter operating time, cost-effectiveness and availability as an outpatient-based procedure. The disadvantage of this procedure is that it is mandatory to have a second retinopexy procedure, such as laser photocoagulation. Furthermore, it requires maintaining a certain posture after the procedure for at least several days or weeks which can be very difficult for old, asthmatic and obese patients. Moreover, missed or new breaks and higher probability of needing a second operation decreased its popularity.

There are some limitations of the study. There was a selection bias as all the respondents were the member of Pakistan Vitreo-retinal Society. Furthermore, not all members of vitreoretinal society responded in the given duration. In addition, the scenarios presented were simple RRD with location of breaks either in upper half or lower half of retina; however, in real world, there are variety of clinical variables in an eye with RRD. Adding on, we did not include questions regarding the complications during or after the surgery, rate of reattachment after first surgery and RRD surgery in children. In future we can have participation of more VR surgeons and this data will be available for comparison.

\section{CONCULSION}

There is an increased trend towards PPV as a primary procedure for RRD. Most of the VR surgeons in Pakistan prefer local anaesthesia over general anaesthesia. In non-PVD RD, SB is preferred by most of VR surgeons. However, in superior RD with PVD, $\mathrm{PPV}$ is considered as a procedure of choice and gas is selected as a preferred tamponade. In inferior RD most of the respondents preferred silicone oil.

\section{Ethical Approval}

The study was approved by the Institutional review board/ Ethical review board. (167-987-2020)

\section{Conflict of Interest}

Authors declared no conflict of interest

\section{REFERENCES}

1. Lumi X, Lužnik Z, Petrovski G, Petrovski BÉ, Hawlina M. Anatomical success rate of pars plana vitrectomy for treatment of complex rhegmatogenous retinal detachment. BMC Ophthalmology, 2016; 16 (1): 216.

2. Feltgen $\mathbf{N}$, Walter $\mathbf{P}$. Rhegmatogenous retinal detachment - an ophthalmologic emergency. Dtsch ÄrzteblInt. 2014; 111 (1-2): 12.

3. Nemet A, Moshiri A, Yiu G, Loewenstein A, Moisseiev E. A review of innovations in rhegmatogenous retinal detachment surgical techniques. J Ophthalmol. 2017; 2017: 4310643.

4. Hatef E, Sena DF, Fallano KA, Crews J, Do DV. Pneumatic retinopexy versus scleral buckle for repairing simple rhegmatogenous retinal detachments. Cochrane Database Syst Rev. 2015; (5): CD008350.

5. Kuhn F, Aylward B. Rhegmatogenous retinal detachment: a reappraisal of its pathophysiology and treatment. Ophth Res. 2014; 51 (1): 15-31.

6. Norregaard JC, Thoning H, Andersen TF, BernthPetersen P, Javitt JC, Anderson GF. Risk of retinal detachment following cataract extraction: results from the International Cataract Surgery Outcomes Study. Br J Ophthalmo. 1996; 80 (8): 689-693.

7. Ghazi NG, Green WR. Pathology and pathogenesis of retinal detachment. Eye, 2002; 16 (4): 411.

8. Olsen TW, Chang TS, Sternberg P. Retinal detachments associated with blunt trauma. Semin Ophthalmol. 1995; 10 (1): 17-27.

9. Lumi X, Hawlina M, Glavač D, Facskó A, Moe MC, Kaarniranta $\mathbf{K}$, et al. Aging of the vitreous: from acute onset floaters and flashes to retinal detachment. Ageing Res Rev. 2015; 21: 71-77. 
10. Ykoff CC, Schwartz SG, Adelman RA, Brucker AJ, Flynn HW. Primary rhegmatogenous retinal detachment repair: evidence supports an individualised approach. Br J Ophthalmol. 2015; 99 (11): 1451-1453.

11. Reeves MG, Pershing S, Afshar AR. Choice of Primary Rhegmatogenous Retinal Detachment Repair Method in US Commercially Insured and Medicare Advantage Patients, 2003-2016. Am J Ophthalmol. 2018; 196: 82-90.

12. Shinkai Y, Oshima Y, Yoneda K, Kogo J, Imai H, Watanabe A, et al. 27G Vitrectomy Study Group. Multicenter survey of sutureless 27-gauge vitrectomy for primary rhegmatogenous retinal detachment: a consecutive series of 410 cases. Graefes Arch Clin Exp Ophthalmol. 2019; 257 (12): 2591-2600.

13. Awan A. Primary Rhegmatogenous Retinal Detachment Surgery in Modern Era. Pak J Ophthalmol. 2018; 34 (2): 70-73.

14. Chneider EW, Geraets RL, Johnson MW. Pars plana vitrectomy without adjuvant procedures for repair of primary rhegmatogenous retinal detachment. Retina, 2012; 32 (2): 213-219.

15. Vail D, Pershing S, Reeves MG, Afshar AR. The relative impact of patient, physician, and geographic factors on variation in primary rhegmatogenous retinal detachment management. Ophthalmology, 2020; 127 (1): 97-106.

16. Hwang JC. Regional practice patterns for retinal detachment repair in the United States. Am J Ophthalmol. 2012; 153 (6): 1125-1128.
17. PAT survey. ASRS (American Society of Retina Specialists). Available at: https://www.asrs.org/asrscommunity/pat-survey. Accessed 16 September 2020.

18. Fischer CV, Kulanga M, Hoerauf $\mathbf{H}$. Trends in retinal detachment surgery: What has changed compared to 2001? Ophthalmologe. 2018; 115 (8): 669.

19. Cho GE, Kim SW, Kang SW. Changing Trends in Surgery for Retinal Detachment in Korea. Korean J Ophthalmol. 2014; 28 (6): 451-459.

20. Eibenberger K, Georgopoulos M, Rezar-Dreindl S, Schmidt-Erfurth U, Sacu S. Development of surgical management in primary rhegmatogenous retinal detachment treatment from 2009 to 2015. Curr Eye Res. 2018; 43 (4): 517-525.

21. Minihan M, Tanner V, Williamson TH. Primary rhegmatogenous retinal detachment: 20 years of change. British Journal of Ophthalmology, 2001; 85 (5): 546-548.

\section{Authors' Designation and Contribution \\ Muhammad Amer Awan; Consultant Ophthalmologist: Wrote the introduction, discussion, results and references, approved the final version.}

Javeria Muid; Postgraduate Trainee: Helped with discussion, figures and results, Helped with final review of the study, approved the final version. 